
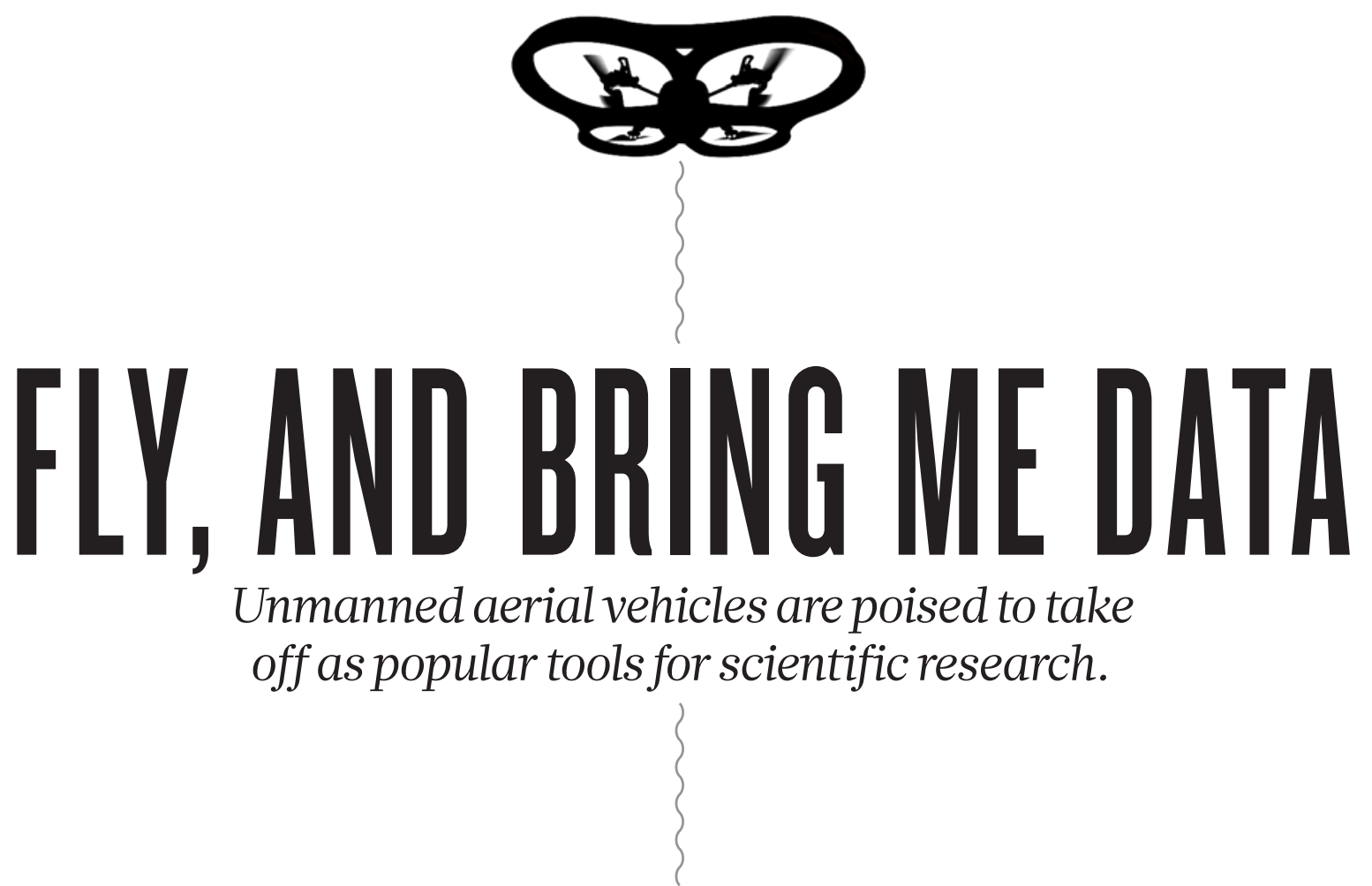

BY EMMA MARRIS

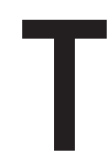

he Tempest - wingspan 3.2 metres, cruising speed 75 knots - was designed to fly into severe storms. But during a test run in March for a new project, it is soaring through the bluest of skies. On the ground below, PhD student Maciej Stachura of the University of Colorado (UC), Boulder, is tapping on a tablet computer, transferring control to the aircraft's own computer after a manual takeoff. Systems engineer James Mack keeps his hands loose around a controller in case a problem arises, while Neeti Wagle, another $\mathrm{PhD}$ student, scans the skies to make sure the Tempest does not collide with anything.

The plane's job today is to locate a beacon sending out a simulated distress signal. As it circles overhead, the Tempest's gas-powered engine makes the distinctive lawnmower-like noise that calls to mind the informal name often given to such aircraft: drones. Unmanned aerial vehicle (UAV) is more commonly used in scientific circles.

The UC Boulder team watches and listens as the 40 minutes or so of flight time tick by and the Tempest becomes a distant speck in the bright sky. Then a note of concern enters Stachura's voice. "It is not doing a great job. It should be getting closer to us at this point," he says. Finally, the drone turns and heads back towards the beacon. "Oh, there it goes," says Stachura, clearly relieved.

The use of drones in science has taken a similarly roundabout route. NASA first experimented with custom-built UAVs in high-altitude research during the 1970s, but unmanned planes have been slow to catch on. Drones with top-notch sensors were too expensive to tempt researchers and cheap versions could not offer much of value. During the past decade, however, lower prices and technical advances - from on-board navigation using the Global Positioning System (GPS) to miniaturization of autopilots - have lured many scientific groups to experiment with UAVs.

Already, they offer an efficient way to gather data and are making important advances in polar research, volcano studies and wildlife biology. "They are on their way to becoming this indispensable and

revolutionary technology," says Adam Watts, an ecologist at the University of Florida in Gainesville who has flown drones for years.

But technical and legal hurdles stand in the way of their wider use. Researchers are trying to improve the autonomy, manoeuvrability and endurance of UAVs. And regulations, particularly in the United States, place strict limits on where and how researchers can use the devices. If these rules loosen up - and there are signs that they may - flying science robots may start taking to the skies in much greater numbers.

\title{
LOFTY HEIGHTS
}

The drones used by military forces to hunt down enemies have attracted growing scrutiny in recent years, but some of them have also been used for science. NASA has conducted hurricane and climate studies with Northrop Grumman's Global Hawk, which can reach an altitude of nearly 20 kilometres - much higher than commercial planes fly. The agency got the drone for free from the Air Force, but interested scientists must be prepared to pay US $\$ 20$ million for such a craft - no sensors included.

Most researchers have to make do with much smaller and cheaper systems. A radio-controlled fixed-wing UAV such as the Tempest can be bought off the shelf for a few thousand dollars. And quadrotor helicopters can be purchased for just $\$ 300$. Slap on a few sensors, an autopilot and a cheap computer preloaded with algorithms, and researchers have an unmanned aerial system (UAS).

Despite the differences in equipment, military and civilian droneresearch programmes have been closely linked, with advances flowing

DNATURE.COM To see robot quadrotors play the James Bond theme: go.nature.com/mmhng4 between the two sides. Many university UAV programmes are, in fact, part-funded by the military.

For now, most researchers working with drones are focused on improving the technology to make the devices more agile, more autonomous and better able to work in groups. Autonomy requires a 


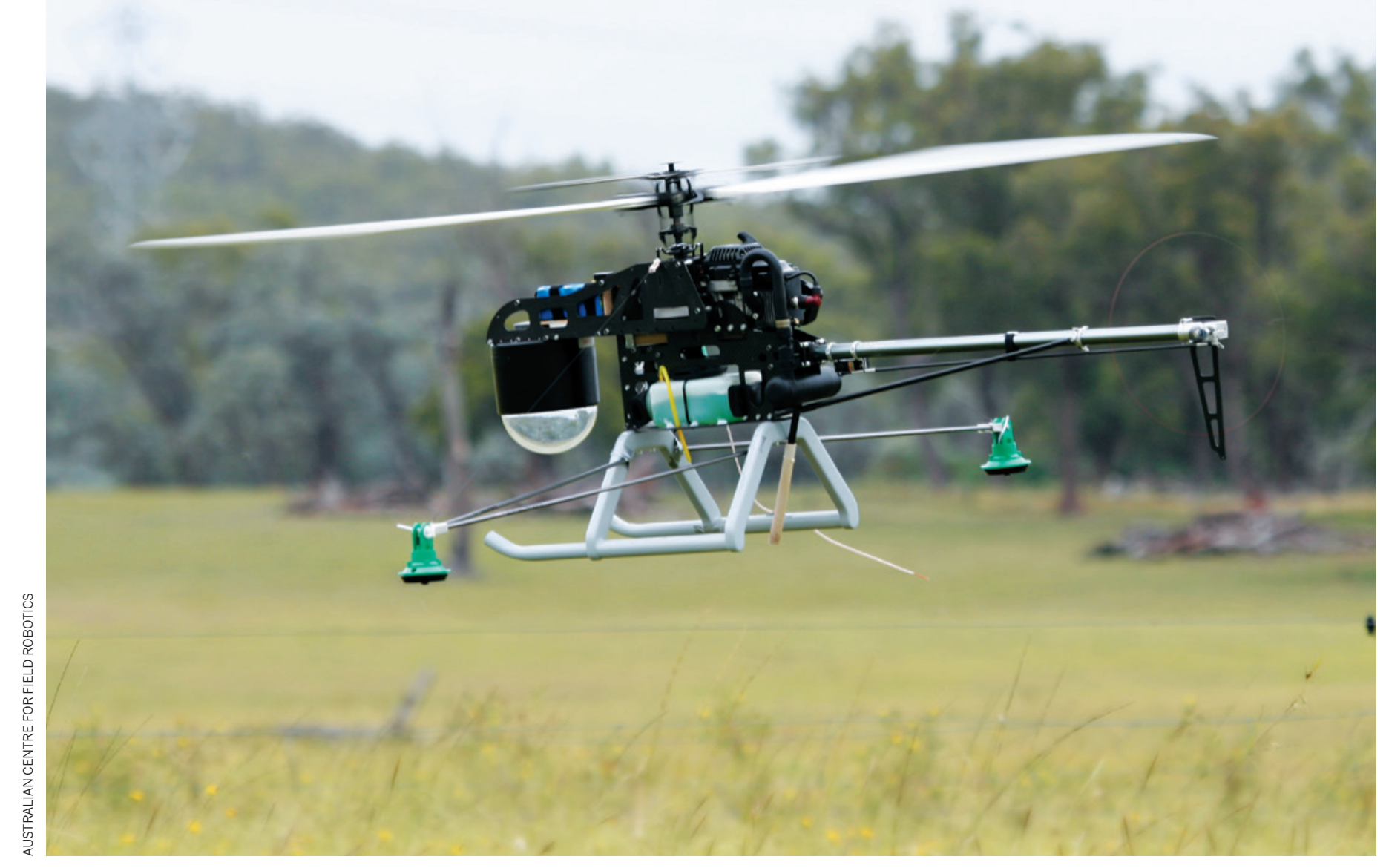

suite of algorithms to interpret data from sensors, make decisions about where to fly, control the plane's path and classify objects captured by the UAV's cameras. And all of that computing has to happen in real time on tiny, light computers bouncing around in three-dimensional space.

One area of focus is vision-based navigation. Systems that rely on the GPS can achieve little better than 3-metre resolution at best - fine for open outdoor landscapes, but not good enough for urban areas or indoor settings. Drone developers would like to send their machines into earthquake-damaged buildings to look for survivors, which would mean avoiding errant beams, power lines and closed windows. To do this, an aircraft requires a complex system of cameras, gyroscopes and accelerometers to figure out where it is - and where the obstacles are.

A team led by Ashutosh Natraj, now at the University of Oxford, UK, has taught drones with fish-eye cameras how to 'find' themselves. The robots' algorithms divide up the circular visual field into sky and ground, identify a horizon line between the two and then derive the drone's altitude and orientation. For city flying, the team is writing algorithms that recognize and use the verticals and horizontals of buildings and streets as guides to navigate up and down, forwards and backwards. At night, the drone could project a laser pattern onto its surroundings to orient itself. Camera-based navigation is smart, says Natraj, because a single camera collects more quality information than a number of expensive, heavy sensors such as laser range-finders, obviating the need to integrate many sensors. As part of a three-year project to design UAVs that can deliver medical care after natural disasters, Natraj is developing systems to do all the image processing on-board the helicopter, rather than through a wireless connection to a separate computer.

The Oxford disaster-relief UAV project is hoping to develop multiple UAVs that talk to one another. Such research on swarms is a hot area, says Hyunchul Shim, director of the Center of Field Robotics for Innovation, Exploration and Defense at the Korea Advanced Institute of Science and Technology in Daejeon, South Korea. "If you go fast, go
An autonomous research drone in Australia sprays herbicide onto weeds. dancy in case some machines fail. But the use of more vehicles also adds complexity. Drones working together have to be able to communicate with one another and make collective decisions.

Researchers are also focusing on increasing the endurance of UAVs, most of which are fuelled by gas engines and batteries. To keep the weight and costs down, researchers often use tiny drones with limited fuel capacity, which means short flights. Some groups are working on miniaturizing batteries, others on making the planes smart enough to take advantage of thermal updrafts and wind features, as birds and gliders do. Roland Siegwart, head of the Autonomous Systems Lab at the Swiss Federal Institute of Technology Zurich, has a team developing solar-powered planes that would never have to land. "I call them 'lowflying satellites"' he jokes. They could actually work better than satellites for collecting data, because researchers could direct them. "You can have an up-to-date image of bush fires, move them over illegal logging operations or look for people lost in the ocean," Siegwart says.

\section{VIDEO STARS}

Teams working on UAVs tend to keep abreast of each other's work through videos posted online. The field's biggest YouTube 'star' is Vijay Kumar at the University of Pennsylvania in Philadelphia. Kumar's group controls quadrotor helicopters indoors with a modified Vicon system the motion-capture system used in Hollywood and by the video-game industry. His videos show drones flying in tight formation, transporting two-by-fours, and even - in one video with more than 3 million views performing the James Bond theme on multiple instruments. "The Internet has changed the rules," says Shim. And, Siegwart says, "It also spreads the information a little further, which helps attract good students." 
With new talent helping to make drones smarter and cheaper, the regulations that control unmanned flight will be the biggest barrier to their expanded use in research. "This is still the major issue," says Siegwart.

That is particularly true in the United States, where Federal Aviation Administration (FAA) rules make it laborious to get permission to fly drones outside (except for non-commercial hobbyists, for whom the rules are looser). "We need permission to go out into a field on campus and fly something that is six inches off the ground," grouses Eric Frew, head of the Research and Engineering Center for Unmanned Vehicles at UC Boulder. "It is a one-size-fits-all approach."

The FAA, based in Washington $\mathrm{DC}$, requires that would-be drone operators apply for and receive one of two certificates for their research programme if they want to fly their UAV s outdoors. The applications request a lot of information, "so the FAA can determine if the operation can be conducted without hazard to other aircraft or people and property on the ground", according to the agency's communications office. This means that certifica-

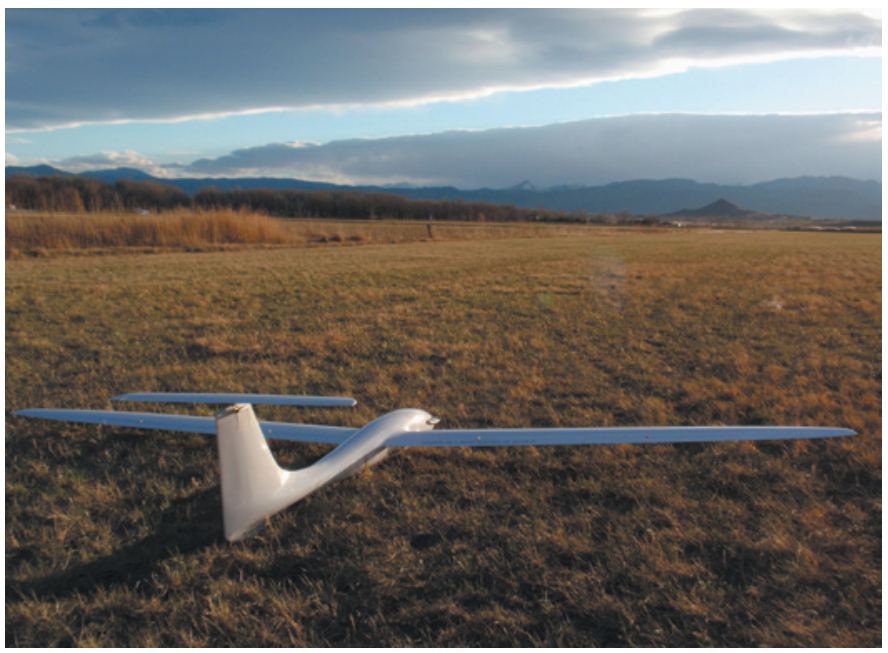

The Tempest in Colorado was designed to collect data from severe storms. updrafts and ash that could choke a plane's engines.

James Maslanik, a remote-sensing expert at UC Boulder, has been involved in a number of studies using drones to measure various qualities of sea ice in the polar regions since 2000 . Here, too, UAVs can venture into regions too dangerous for a manned aircraft. In the Arctic, Maslanik says, "we are flying these things at 100 feet off the ice, wind is 80 knots, temperature of minus $40^{\circ} \mathrm{C}$."

At the opposite end of Earth, researchers from UC Boulder have used UAVs to measure jets of wind that scream down from the Antarctic plateau into Terra Nova Bay. Such measurements could help scientists to understand the dynamics of sea-ice formation around Antarctica, which creates dense salty water that sinks and helps to drive global ocean currents. "Nobody had an aircraft out there during winter when the winds are strongest and took measurements because the conditions are too extreme," says Maslanik. The data collected so far, he says, show unexpectedly complex wind patterns, including fierce, localized jets that push sea ice off shore and speed up its formation.

Biologists are also starting to use UAVs in their field work. tions are not granted for flights in cities and other crowded areas. These certifications are also limited to a 20-mile-square area (around 32 square kilometres), so when the UC Boulder team took Tempest storm-chasing across swathes of the country, they needed 59 separate permissions.

Once a certification is obtained, usually within 60 days, a group can fly its aircraft during daylight hours in the designated spot for a year or two as long as they file a NOTAM — a Notice to Airmen — in advance with the FAA every time they want to fly.

Each flight also requires a certified pilot. During the March test flight, that was Stachura, who spent most of the test staring into the plane's controls on his tablet. The FAA also requires that an observer be on hand to watch for potential collisions and that someone be monitoring the radio from the local airport.

\section{FLIGHT DELAYS}

Eric Johnson, who studies UAVs at the University of Georgia in Athens, has looked at regulations around the world and says that "among NATO countries, the United States is about the worst". But as long as there are no accidents, the consensus seems to be that the regulations will loosen. The FAA Modernization and Reform Act, which passed last year, calls for the US Department of Transportation to produce a plan by late 2015 for "the safe integration of civil unmanned aircraft systems into the national airspace system".

By contrast, says Johnson, Australia and Canada allow the most types of operation, perhaps because both countries have a lot of airspace and smallish bureaucracies. Salah Sukkarieh, who studies robotics and intelligent systems at the University of Sydney in Australia says that the country's liberal regulations are allowing the UAS field to grow there, despite its funding being a fraction of that available to US scientists.

Although most drone research has focused on improving the UAVs themselves, some scientists have been putting the devices to use. In March, NASA used a small electric military drone, the Dragon Eye, to sample and photograph the noxious gas plume spewing from Turrialba Volcano near San Jose in Costa Rica. The team compared the Dragon Eye's measurements of sulphur dioxide to those made by the Terra satellite in an effort to calibrate the space-based readings. It would have been too risky to send a human pilot near the volcano, where there are strong
In India, the conservation group WWF is using drones to look for rhino poachers. Tom McKinnon, a retired engineer and managing director of InventWorks, a product-development firm in Boulder, is outfitting autonomous helicopters with nets to capture rare Mongolian vultures so that scientists can attach transmitters and study their movements.

On the plant side, Sukkarieh has developed a system using a fixedwing UAV and a helicopter in tandem to locate weeds in remote rangelands and spray them with herbicide. And several groups are teaching drones how to tell one kind of plant from another, so that they can make maps of vegetation. Rather than purchase advanced sensors, which add weight and increase costs, Sukkarieh's team is writing code to allow the UAV to map and classify vegetation using just GPS, a camera and an inertial measuring unit, which collects data on the position of the aircraft in space. The challenge of making trade-offs between sensors and weight has prompted Sukkarieh to think about designing UAV systems from scratch around their specific tasks, rather than just bolting sensors to an off-the-shelf aircraft. "What if the wings were sensors themselves?" he wonders.

For researchers without engineering expertise, however, the available UAV s offer plenty of opportunities. The Scottish Environmental Protection Agency, for example, purchased a drone in 2012 from Swiss company senseFly to survey estuaries for algal blooms - something that is difficult to do on foot. Susan Stevens, a scientist at the agency, says "you can get involved and use the technology without being an expert in it".

Still, the best landings come with experience. As the UC Boulder team finishes up testing the Tempest, Mack, who was a UAV hobbyist before he joined this research team, gently sets the drone down on its belly in a patch of short grass. He picks it up in one hand to carry it back to the van.

Everyone is relaxed, having spent most of the 40-minute test flight doing little more than watching the Tempest and enjoying the spring day. If this is the future of field research, it looks pretty easy. "If everything goes well, it is fairly boring," Stachura acknowledges. "Because it is autonomous, right?"

Emma Marris is a freelance writer in Klamath Falls, Oregon. 